\title{
Deciphering the Interaction of a Macrocyclic Tetranuclear Zn(II) complex with Biomimicking Surfactant: A Case Study using Cetyltrimethylammonium Bromide
}

\author{
Anwita Sarkar ${ }^{1(\mathbb{D})}$, Sristi Raj Rai ${ }^{1(\mathbb{D})}$, Megha Sen Chowdhury ${ }^{2(\mathbb{D})}$, Tonmoy Chakraborty ${ }^{3(\mathbb{D}}$, Sanchari \\ Dasgupta $^{3(\mathbb{D})}$, Debasis Das ${ }^{3(\mathbb{D})}$, Abir Bhattacharya ${ }^{4}(\mathbb{D})$, Madhumita Mukhopadhyay 2,*(D), Debosreeta \\ Bose $2, *$ (D)
}

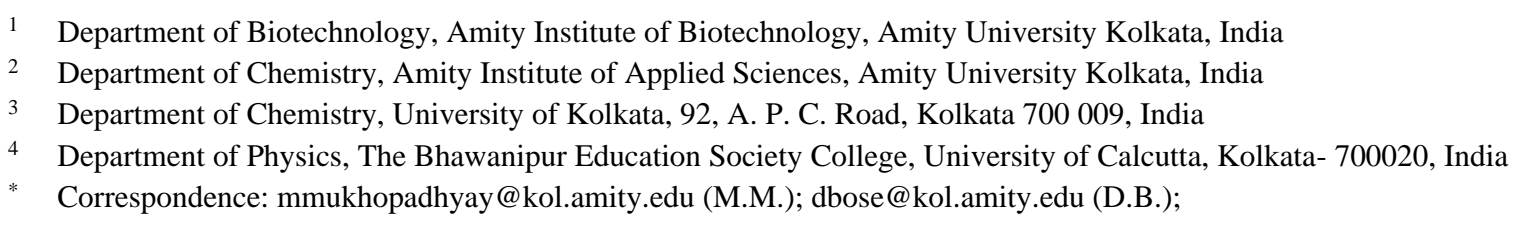

Received: 25.03.2021; Revised: 10.05.2021; Accepted: 14.05.2021; Published: 18.06.2021

\begin{abstract}
The detection of various biologically important cationic and anionic species has led to the synthesis of various Zinc (II) Schiff base complexes, which bear a large application in catalysis and sensors. A recently reported study has indicated a synthesis of a 48 membered macrocyclic $\mathrm{Zn}$ (II) Schiff base complex, which functions as a dual anion sensor (DAS) containing a protonated amine group. In the present investigation, an attempt has been made to study the interaction of the $\mathrm{Zn}$ tetranuclear complex (Zn-TCom), a neutral complex, with a cationic surfactant cetyltrimethylammonium bromide (CTAB), employing steady-state absorption and fluorescence studies. The change in the photophysical properties of the fluorophore is studied on binding with the surfactant. The steady-state absorption and emission studies reveal that the polarity of the surrounding of the fluorophore has been modified upon binding with CTAB. A study on the alteration of polarity during the interaction of Zn-TCom with the surfactant is studied by comparing the fluorescence behavior of the probes in a micro heterogeneous environment in a mixture of dioxane and water of varying composition. The binding constant magnitude (analyzed using the Hildebrand equation) manifests that the Schiff base binds to the micellar compartment. These results are manifested to enhance the potential application of the macrocyclic complex in the field of biochemistry. Furthermore, quantum chemical calculations are undertaken to correlate the molecular structure of Zn-TCom with the associated frontier molecular orbitals. The parameters like the energy of HOMO, LUMO and the associated Mulliken charges with respect to individual elements are computed using the single point geometry. The studied reports are correlated with the feasibility of binding with bio mimicking environment or bio proteins for selective application as drug carriers.
\end{abstract}

Keywords: absorption; binding constant; fluorescence; micelle; Schiff base.

(C) 2021 by the authors. This article is an open-access article distributed under the terms and conditions of the Creative Commons Attribution (CC BY) license (https://creativecommons.org/licenses/by/4.0/).

\section{Introduction}

A microenvironment is the immediate small-scale distinct surroundings of the larger environment in any chemical or biological system that gets affected as a result of various activities. In fact, the micro changes within the environment are mainly responsible for the 
overall behavior of the larger ensemble/cluster. Organized assemblies/Microheterogeneous environments have witnessed immense growth in the last three decades owing to their structural similarity with real biomembranes [1]. Monitoring the changes in these microheterogeneous environments can help us understand the behavior of a chemical complex in altering situations. Detection of ionic species in a particular environment is necessary owing to the presence of unwanted ions (cations/anions) or to analyse the changes associated with the concentration alteration of the system. However, such changes in the biological system can prove to be toxic. Amines or synthesized polyamines can be widely used as anion receptors as they are usually protonated, thus charged in neutral and acidic aqueous solutions [2]. These imine complexes bear extensive application in the medicinal industry owing to their antibacterial, antifungal, antiviral activities. The recent articles on the binding interaction of the bioactive fluorophores with the surfactants throw considerable light on the bioefficacy of the fluorophores [3]. In today's date, Chakraborty et al. has synthesized \& characterized an abiotic receptor to detect the presence of anions using a 48-membered macrocyclic $\mathrm{Zn}$ (II)-Schiff base complex that acts as a dual anion sensor (DAS) and contains protonated imine [4]. For such a system, a probable pathway has been established upon combining experimental and theoretical approaches wherein the abstraction of such iminium proton by the anions $\left(\mathrm{F}^{-}\right.$and $\left.\mathrm{AcO}^{-}\right)$results in significant color change. Figure 1 represents the ball and sticks model of the tetrameric Zn (II) Schiff base along with the asymmetric unit [4]. Complexes like Schiff base have a wide range of applications in the area of catalysis, sensing, etc. [3, 5-9] due to their cost-effectiveness. These bases are said to be good fluorophores due to the extended conjugation that takes place in the ligand backbone [3,5-9]. Their synthetic flexibility, excellent chelating ability, and easy excretion tenability through masking and demasking feature are of great importance. The DAS is reported to have high selectivity for both fluoride $\left(\mathrm{F}^{-}\right)$\& acetate $\left(\mathrm{AcO}^{-}\right)$anions in methanolic solutions. Fluoride is used in a number of drugs, dental care and also required for osteoporosis treatment [10-16].

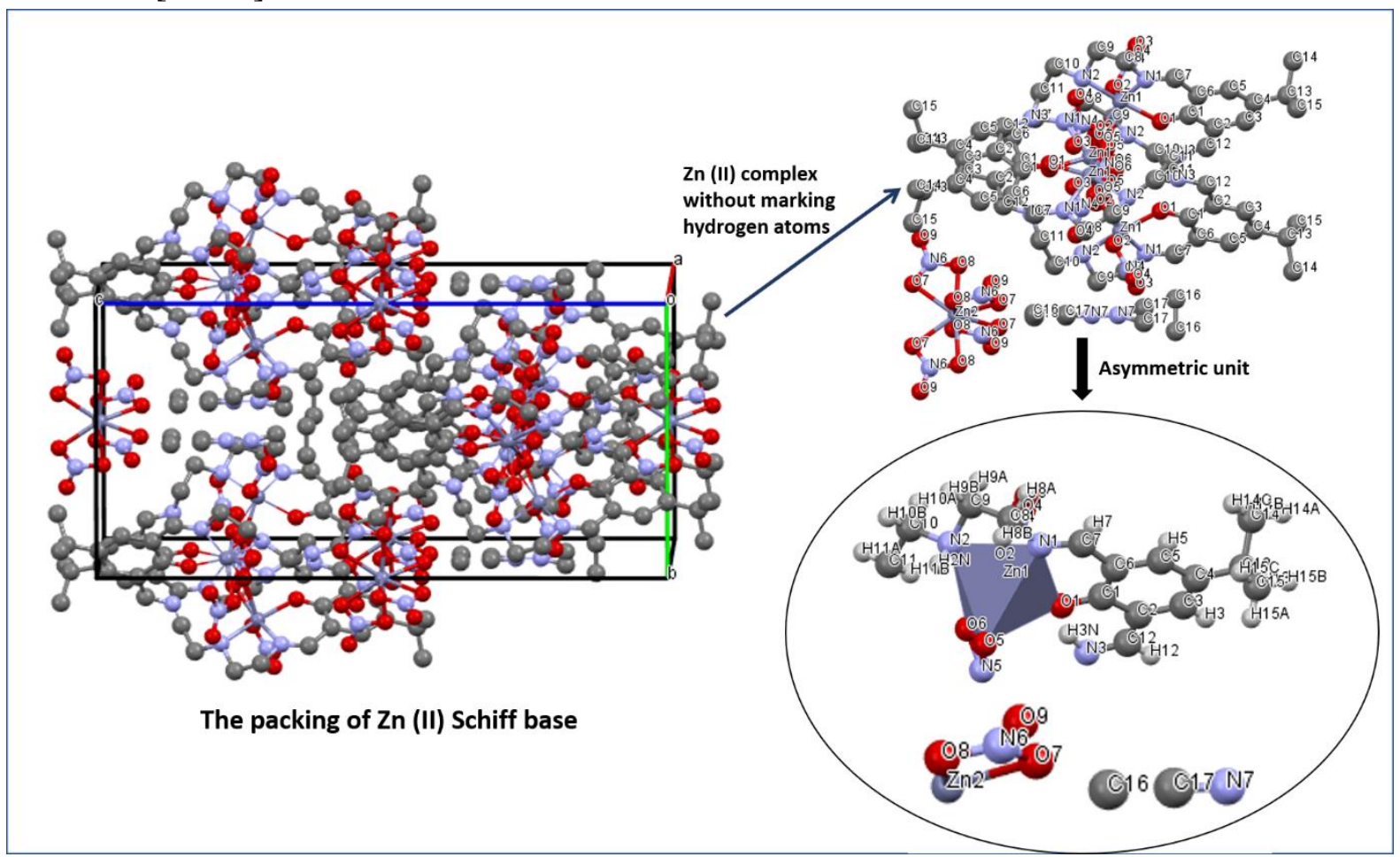

Figure 1. The packing of $\mathrm{Zn}$ (II) Schiff base along with the ball and stick model of the asymmetric unit. 
It is important to check for the content of $\mathrm{F}^{-}$ion in the human body as an increase in ingestion amount can prove to be toxic. Hyper fluorine content can cause fluorosis, decreases thyroid's activity, bone disorders, urolithiasis, stomach ulcers and cancer [17-20]. Acetate ions are also found in the human body in the form of acetyl coenzyme A form that helps in energy production via the Krebs cycle [21]. Studies reveal that a higher quantity of sodium acetate can induce headaches in sensitized rats [22]. Moreover, the DAS can also be commercially used to detect $\mathrm{F}^{-}$in toothpaste and solid-state detection of both the anions. In this mentioned background, the biochemical reactions are affected by the labile chemical reactions, affecting the microenvironment directly in both ex-situ biological media for in-vivo experiments.

Therefore, studying the underlying interactive chemistry with the fluorophore molecules of the zinc tetranuclear complexes in organized biomimicking/microheterogenous media is an important aspect of modern research. Among numerous Schiff base(s), Zn (II) based functional compounds are rare but pose significant photophysical properties which enable ease in detailed analyses. The literature report reveals that the X-ray analysis of a single crystal of the tetranuclear complex cation $\left[\mathrm{Zn}_{4}\left(\mathrm{LH}_{3}\right)\left(\mathrm{NO}_{3}\right)_{5}\right]^{2+}$ is counterbalanced by $\left[\mathrm{Zn}\left(\mathrm{NO}_{3}\right)_{4}\right]^{2-}$ anion [4]. The primary novelty of the zinc tetranuclear complex ( $\mathrm{Zn}$-Tc, empirical formula $=\mathrm{C}_{62} \mathrm{H}_{84} \mathrm{~N}_{22} \mathrm{O}_{31} \mathrm{Zn}_{5}$ ) is that it can detect these two mentioned ions even in a mixture of anions $\left(\mathrm{Cl}^{-}, \mathrm{I}^{-}, \mathrm{S}^{2-}, \mathrm{PO}_{4} 3-, \mathrm{S}_{2} \mathrm{O}_{3}{ }^{2-}\right)$. For the functionalization and performance evaluation of the synthesized Schiff base sensors, fluorescence study of such compounds with and without a binding agent(s) poses an important arena for selective application. In order to suit such a study, the synthesized base needs to be fluorescence active either in its pristine form or by insertion of an active fluorophore agent [23]. The colorimetric study of this $\mathrm{Zn}$ (II) compound reveals the change in color from bright yellow to pale yellow (under the naked eye) and from green to blue (under UV lamp) with considerable spectral change in the presence of $\mathrm{F}$ and AcO, respectively in aqueous methanolic solution [4]. In such an aspect, micelle application plays an important role in acting as the model biomimicking system for screening such Schiff bases to be effective drug carriers or applications [23-26]. Therefore, the fundamental research reported in the present context is based on the interaction among micellar aggregation with $\mathrm{Zn}$ (II) Schiff base fluorophore. The primary factors governing such interaction process are optimized using critical micellar concentration (CMC) and the critical temperature termed Kraft Temperature [27]. The prime area of research lies in the interaction of target $\mathrm{Zn}$ (II) compound with the layers of the micelle viz. non-polar interior or core of the micelle (containing the hydrocarbon chains of the surfactants), the Stern layer (formed at the micelle-water interface) for ionic micelle that surrounds the core (for non-ionic micelle, the layer is known as palisade layer), and the wider Gouy-Chapman layer containing the counterions [28,29]. The present report also establishes the alteration of the polarity of the system introducing the probe (compared to that of the bulk aqueous solution) at different concentrations through a micropolarity study. In addition, such a study is correlated in the presence of a water dioxane calibration system. Quina et al. reported the mechanism of photo processes in numerous micro aggregates [30]. The influence of micellar aggregation on kinetic and thermodynamic aspects of different photophysical processes has been studied by Ramamurthy et al. [31]. The authors herein have reported the fluorescent behavior of experimental Zn (II) Schiff base using cetyltrimethylammonium bromide (CTAB), which provides an initial knowledge to decipher the compound in biomembranes [4]. Entrapment of such experimental molecules with variable biomimicking environments explores the potential usefulness of its fluorescence properties to understand its interactions with appropiate biological media. In the present context, a 
comprehensive photophysical study of zinc tetranuclear complexes is reported by utilizing CTAB being the membrane which acts to be biomimicking. Steady-state absorption and emission studies have been reported, which further enables the application of such optimized biocompatible functional materials towards future study regarding interaction with bio protein and application.

\section{Materials and Methods}

The 48 membered macrocyclic compounds of Zn (II) Schiff base complex containing protonated imine is synthesized and reported [4]. Analytical grade CTAB and solvent acetonitrile and 1,4 dioxane (SRL) were used as received along with triply distilled water. The absorption and emission spectral studies were conducted by the Hitachi U-2910 spectrophotometer and Hitachi F-4600 fluorescence spectrophotometer. The concentration of the compound used for all the experiments was kept at $2 \mu \mathrm{M}$. UV sol 4.2 was used for the absorption spectrophotometer and FL sol 4.0-4600 software was used for the fluorometer. An ambient temperature of $303 \mathrm{~K}$ was maintained for performing all the experiments. In order to check the reproducibility, all undertaken photometric characterizations were performed on three sets of the same experimental sample. The experimental sample was subjected to a micro polarity study using fluorescence behavior of the probes in a micro heterogeneous environment in a mixture of dioxane and water of varying composition. The use of a dioxane/water mixture covers a much wider range of polarity and is free from difficulty.

\subsection{Computational details.}

The computational calculations were carried out in the Gaussian 09 suite of the quantum chemical program package [32]. The ground state single point energy of DAS was computed using density functional theory employing 6-31G* basis set. Using Becke's three-parameter hybrid functionals (B3), with nonlocal usage of Lee-Yang-Parr, LYP, (commonly referred to as B3LYP), the structural and energy parameters were calculated. The visualization of the different structural forms of DAS was done by GaussView 5.0 software. The HOMO and LUMO were generated from the computed chk. point files of DAS and visualized through the GaussView 5.0 software. Electronic indices include the energy of the lowest unoccupied molecular orbital (ELumo), energy of the highest occupied molecular orbital (Еномо) of the experimental system viz. Zn-TCom is also computed. The distribution of electronic charge with respect to the elements in the Schiff base is also computed.

\section{Results and Discussion}

\subsection{Steady-state absorption and fluorescence.}

The absorption and emission spectra of the Zn (II) Schiff base complex undertaken in the present context along surface with a cationic surfactant, cetyltrimethylammonium bromide (CTAB) are shown in Figure 2 and 3, respectively. The details of sample identification with respect to the concentration of the surfactant are shown in Table 1 [Zn-TCom: Zinc (II) tetrameric complex]. The aqueous solution of the CTAB-Zn-TCom shows a broad absorption energy band with a maximum at $390 \mathrm{~nm}$ (marked in Figure 2b), which subsequently gets shifted on the addition of the cationic surfactant CTAB. The gradual addition of CTAB to the aqueous solution shows a redshift of the absorption maximum to $400 \mathrm{~nm}$. The redshift in the absorption 
spectrum suggests an occurrence of a destabilized ground state of Zn-TCom in the presence of $\mathrm{CTAB}$ and also reflects a lowering in the polarity of the micellar solution from the aqueous medium.

Table 1. Specification of experimental sample and sample ID

\begin{tabular}{l|l|l} 
Sample ID & Concentration of CTAB surfactant (mM) & Sample ID w.r.t. solution concentration
\end{tabular}

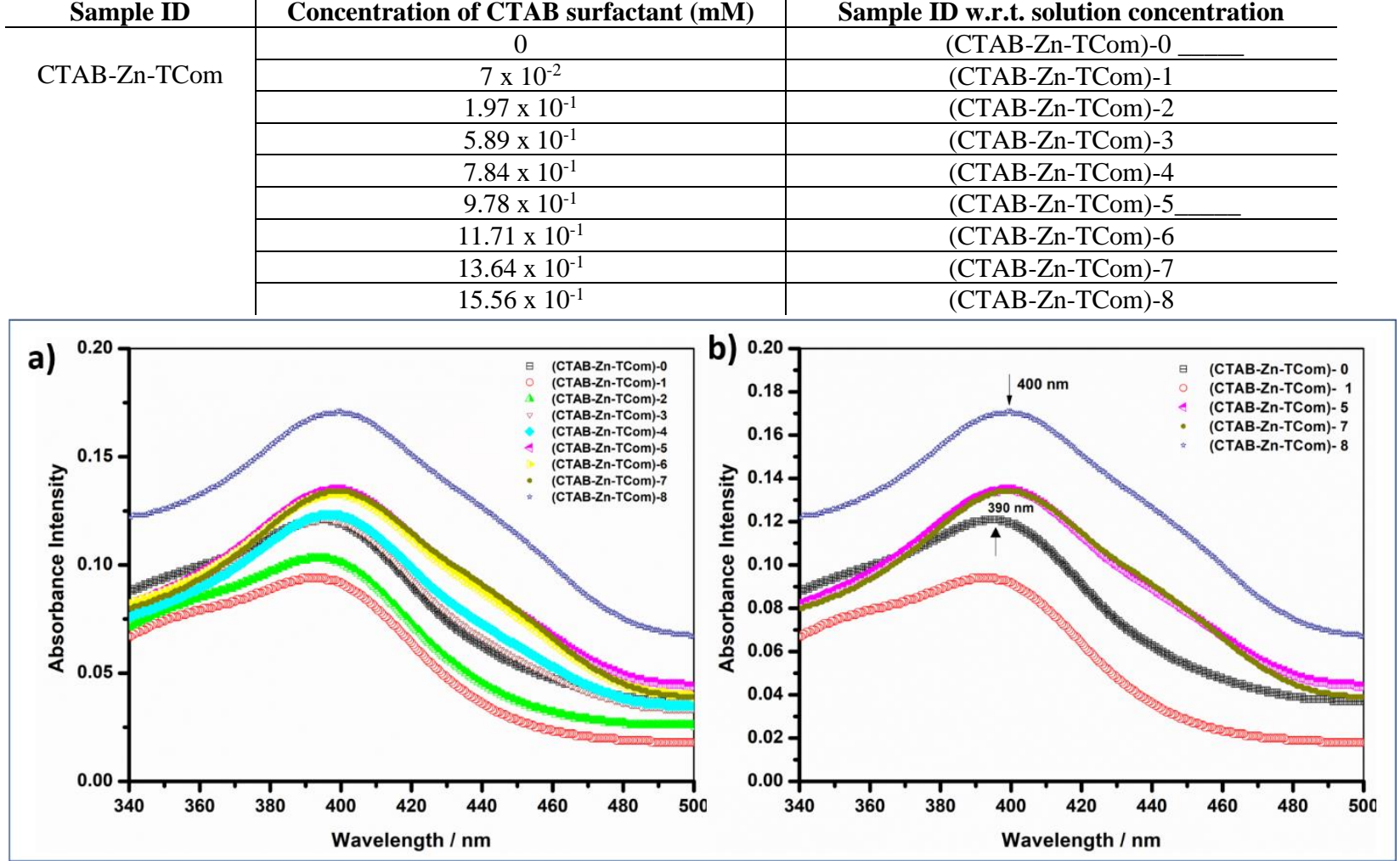

Figure 2. Absorption spectra of CTAB-Zn-TCom: (a) as a function of surfactant (CTAB) concentration; (b) for a selective concentration of CTAB for visualization of redshift.

The lowering in polarity of the parent system upon the addition of CTAB is also analyzed through a micropolarity study in the subsequent sections. Emission spectra of the $C T A B-Z n-T C o m$ are shown in Figure 3. The emission spectra are found to exhibit broadband with a maximum of $495 \mathrm{~nm}$. It shows an unstructured charge transfer (CT) emission band arising from the $\pi \rightarrow \pi^{*}$ transition [33]. The gradual addition of CTAB results in the blue shift of the fluorescence emission spectra. A variation in the fluorescence intensity of the complex as a function of the surfactant concentration is observed. With the addition of CTAB, there is a huge enhancement in the fluorescence intensity up to the saturation limit.
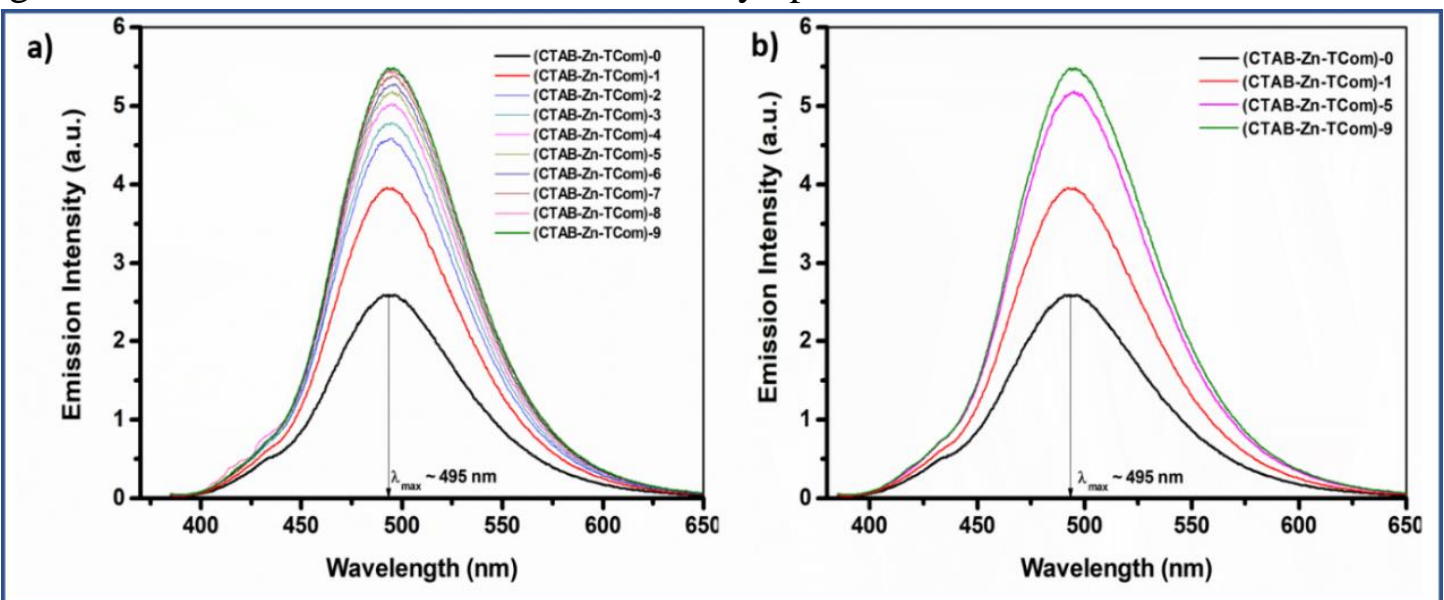

Figure 3. (a) Emission spectra of Zn-TCom solution as a function of variable surfactant (CTAB) concentration; (b) selected composition. 
After attaining the CMC value, the huge enhancement in the emission yield reflects the difference in the microenvironments around the fluorophores in the CTAB media from the pure aqueous phase. The blue shift reflects a less polar environment around the fluorophore than that of the bulk water. The exhibited blue shift indicates the fluorophore interaction with the surfactant, which means the target Schiff base system is interacting affirmatively with the biomimicking environment.

Selected compositions of the CTAB-Zn-TCom are shown in Figure 3b, which clearly exhibit the change in the emission spectra with the subsequent addition of the biomimicking agent, CTAB.

It is found that the emission maximum of the dye shifts towards blue on increasing the percentage of dioxane. A representative plot monitoring the energies corresponding to the fluorescence maximum of the experimental Zn-TCom Schiff base complexes in the waterdioxane mixture against $\mathrm{E}_{\mathrm{T}}(30)$ is depicted in Figure 4. The plot establishes a linear correlation between the two parameters. Interpolating the values of the energies corresponding to the maximum emission values of the $\mathrm{Zn}$ (II) Schiff base complexes bound to BSA at its saturation concentration on the calibration line, the micropolarities around the probes have been determined to be 46.5 for CTAB-Zn-TCom-9. The micropolarity values suggest that at the saturated concentration of the surfactant CTAB, the Zn-TCom becomes less polar.

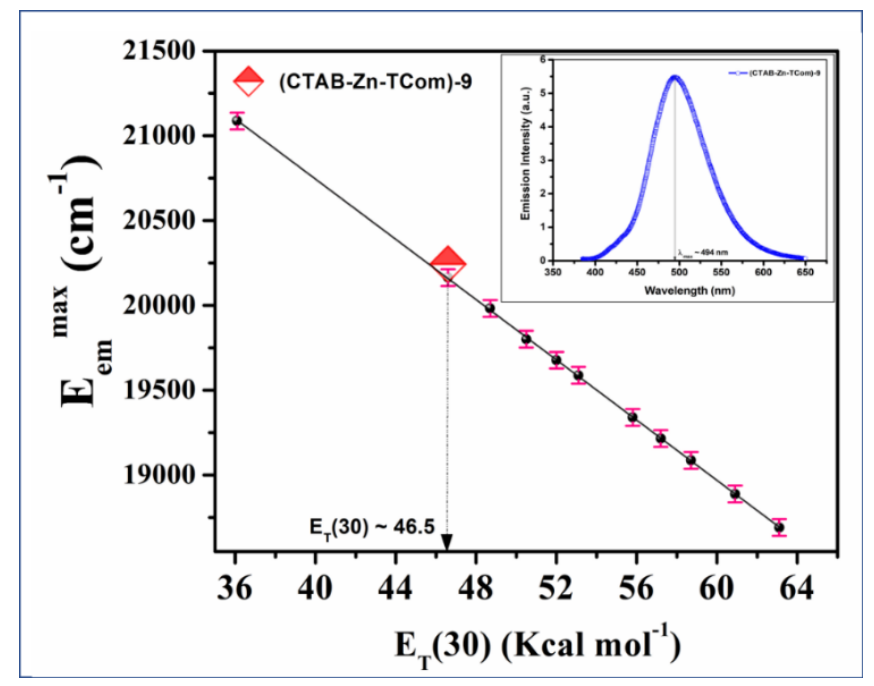

Figure 4. Variation of energies corresponding to the emission maximum ( $\mathrm{E}_{\mathrm{em}}{ }^{\mathrm{max}}$ ) of the $\mathrm{Zn}$ (II) Schiff base complexes with CTAB (CTAB-Zn-TCom-9) in dioxane-water mixture against $\mathrm{E}_{\mathrm{T}}(30)$. The circles give the interpolated $\mathrm{E}_{\mathrm{em}}{ }^{\text {max }}$ values obtained in the $\mathrm{CTAB}$ environment. The respective emission spectra for maximum BSA concentration are shown in the inset of the figure.
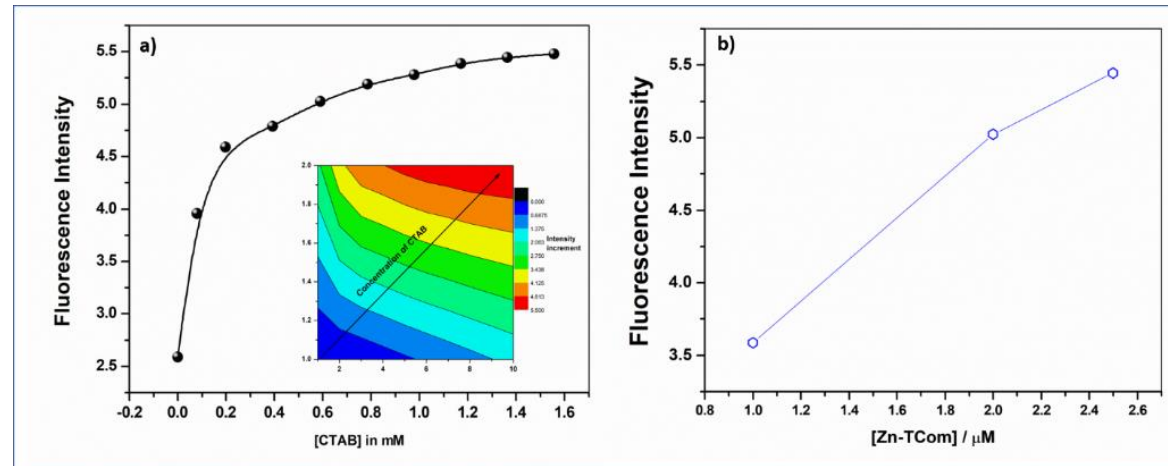

Figure 5. (a) Variation of fluorescence intensity as a function of surfactant concentration [CTAB]; the respective contour map with respect to the intensity is given in the inset of (a); (b) dependence of fluorescence intensity on the concentration of Schiff base complex Zn-TCom. 
This observation of Figure 4 is in coherence with the trend of the emission plot shown in Figure 3 to explain the photophysical properties of $\mathrm{Zn}$ (II) complexes.

The steady increment of fluorescence intensity is observed as a function of surfactant concentration in Figure 5a. The graph suggests an initial escalation in the intensity till $0.2 \mathrm{mM}$ $\mathrm{CTAB}$, after which the rate of intensity increment seems to become sluggish and saturate till the final concentration of $1.6 \mathrm{mM}$. The trend of this dependence can be visualized better in the color contour map shown in the inset figure. The segregated gradation in color demonstrates the depicted variation in fluorescence intensity as a function of concentration. On a similar note, variation of the concentration of Schiff base complex, Zn-TCom, also provides variation in the intensity of the fluorescence intensity. The present context only shows the results using once the specific concentration of the $\mathrm{Zn}$ compound. Since Figure $5 \mathrm{~b}$ reflects a steady increase in the intensity with a change in the concentration of the compound, the selection of any specific concentration within this range effectively explains the photophysics upon combining with the biomimicking agent like CTAB.

\subsection{Probe-micelle binding and correlation with the Frontier Molecular orbital concept.}

The binding constant of the fluorophore in the cationic micellar environment has been determined to understand the degree of interaction between the fluorophore and the micellar units. The binding constant calculation (for 1:1 probe-micelle complexation)follows the method described by Almgren et al. using the fluorescence intensity data as given in equation 1: [34]

$$
\left(I_{\alpha}-I_{0}\right) /\left(I_{c}-I_{0}\right)=1+K[M]^{-1}
$$

where, Io, Ic, and $\mathrm{I}_{\alpha}$ are the fluorescence intensities of Zn-TCom in the absence of CTAB surfactant, at the intermediate surfactant concentration, and under the condition of complete probe-micelle interaction respectively, $\mathrm{K}$ is the binding constant, and $[\mathrm{M}]$ is the micellar concentration.

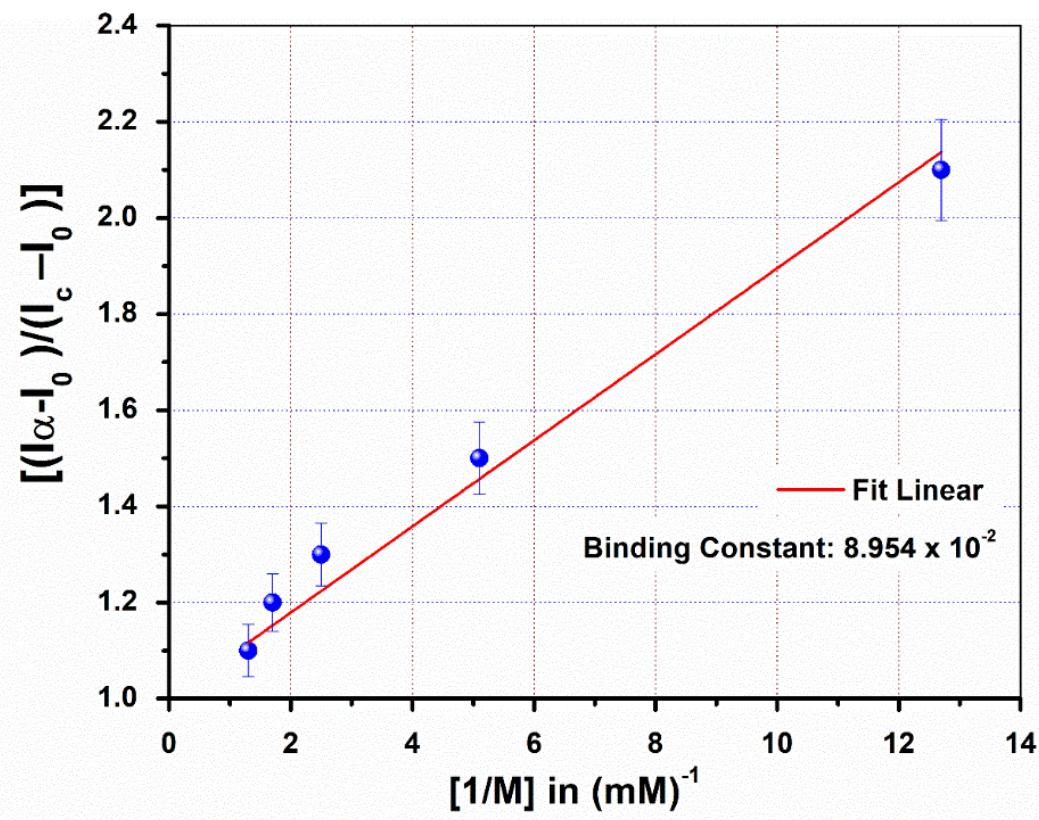

Figure 6. Plot of $\left[\left(\mathrm{I}_{\infty}-\mathrm{I}_{0}\right) /\left(\mathrm{I}_{\mathrm{c}}-\mathrm{I}_{0}\right)\right]$ as a function of surfactant $[\mathrm{CTAB}]$ concentration for the study of the dyeprotein binding interaction. 
The plot for the binding constant is given in Figure 4 along with the respective slope (obtained from fitting the plot). The magnitude of the binding constant for the complex is obtained to be $8.954 \times 10^{-2} \mathrm{mM}^{-1}$. The binding constant corresponds to the interaction between the metal-ligand complexes with the CTAB. It could be inferred that the electronic environment around both the surfactant and the probe exerts a remarkable influence on binding. The magnitude of the binding constant is obtained from the fitting of the plot given in Figure 6, which exhibits the interaction between the dye and the experimental cationic surfactant (CTAB). The graph also establishes the linearity among the intensity ratio and the concentration of CTAB surfactant.

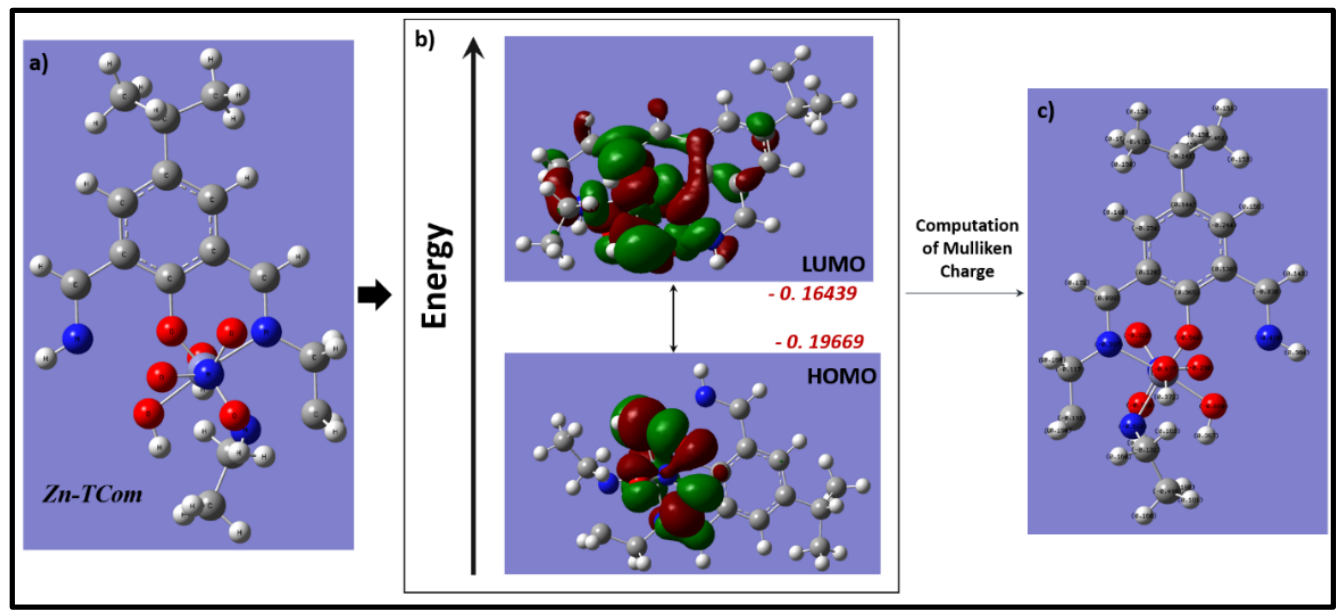

Figure 7. Optimized structure of (a) Zn-TCom and (b) frontier molecular orbitals (HOMO and LUMO); (c) computed Mulliken charges for the system.

Quantum chemical calculations are undertaken to correlate the molecular structure of the present experimental compound viz Zn-TCom, with the associated properties. The frontier molecular orbital (FMO) and the single point optimized structure are elucidated in Figures 7 a and b, respectively. The figure represents the parameters like Elumo, Eномо, $\Delta \mathrm{E}_{\text {Lumo-номо and }}$ the Mulliken charges shown in Figure 7c. The adsorption centers within the molecule which are responsible for interaction during the course of reaction or during association with biomimicking / bio protein entities can be predicted from the FMO. In the present context, the $\mathrm{HOMO}$ is found to be distributed over the tetranuclear central $\mathrm{Zn}$ (II) complex cation of $\left[\mathrm{Zn}_{4}\left(\mathrm{LH}_{3}\right)\left(\mathrm{NO}_{3}\right)_{5}\right] .{ }^{2+}$ For simple understanding, one $\mathrm{Zn}$ site is considered in the optimized geometry as shown in Figure 7a. However, the LUMO corresponds to the complex cation and extended to the cyclic systems in the neighborhood. The present tetranuclear cation is counter balanced by a $\left[\mathrm{Zn}\left(\mathrm{NO}_{3}\right)_{4}\right]^{2-}$ anion, making the macrocyclic ligand negatively charged, thereby posing coherent interaction with the cationic surfactant species. The corresponding energies of HOMO and LUMO are also shown in Figure 7b. The significant negative energy of the HOMO supports the higher stabilization of the compound with respect to the LUMO. It is to be noted that, according to the reviews, the present system enables electron donation (as per high negative HOMO). The bandgap, i.e., among the highest occupied and lowest unoccupied orbital, is the prime factor in defining the molecular electrical transport properties. It may be stated that the energy corresponding to HOMO is correlated with ionization potential, whereas the LUMO energy content is proportional to the electron affinity parameter. Figure $7 \mathrm{c}$ shows the Mulliken population yield towards understanding charge distribution for the present experimental compound. The highest negative charge is localized at the oxygen atom present in the vicinity of the central $\mathrm{Zn}$ (II) system. For simplicity, only one unit of $\mathrm{Zn}$ (II) is considered 
among the tetranuclear species. However, a discrepancy is observed in the localized computed charge in the other five oxygen atoms (as shown in the red color ball and stick model). This indicates the significant influence of the central metal ion towards the electronic contour of the adjacent local electronic environment. Similar perturbation in the local electronic cloud is expected in the vicinity of other $\mathrm{Zn}$ (II) core as well. Such polarization within the Schiff base enables strong interaction with foreign guests like surfactant or bio protein. These analyses are interesting and important in applying such biocompatible Schiff bases as target compounds or in a similar research arena.

\section{Conclusions}

The conclusive details of the present research work include the interaction of the tetranuclear complex of $\mathrm{Zn}$ (II) [Zn-TCom] with biomimicking surfactant like cetyltrimethylammonium bromide (CTAB). Preliminary research reported the effectivity of the tetranuclear compound as an efficient sensor for anions like fluoride and acetates. The relevant experimental studies are also carried out and are reported. The binding interaction of the $\mathrm{Zn}$ TCom complex with CTAB is reported in this work. The photophysical behavior is modified upon binding with the biomimicking environment. Absorbance and Fluorescence studies suggest a decrease in the polarity of the environment surrounding the probe. This suggests that the complex binds with CTAB with a great affinity. A study on the alteration of polarity during the interaction of $\mathrm{Zn}$-TCom with the surfactant is studied upon a comparison of the fluorescence behavior of the probes in a micro heterogeneous environment in a mixture of dioxane and water of varying composition. The binding constant of (0.0089) magnitude (analyzed using Hildebrand equation) manifests that the Schiff base binds to the micellar compartment. Furthermore, quantum chemical calculations are undertaken to correlate the molecular structure of Zn-TCom with the associated frontier molecular orbitals. The parameters like the energy of HOMO, LUMO and the associated Mulliken charges with respect to individual elements are computed using the optimized geometry. The findings are correlated with the feasibility of binding with the biomimicking environment or bio proteins for selective application as drug carriers.

\section{Funding}

This research received funding from Amity University, Kolkata.

\section{Acknowledgments}

The authors from Amity University, Kolkata, acknowledge the financial and structural support provided by the university.

\section{Conflicts of Interest}

The authors declare no conflict of interest.

\section{References}

1. Jin, M.-Z.; Jin, W.-L. The updated landscape of tumor microenvironment and drug repurposing. Signal Transduction and Targeted Therapy 2020, 5, 166, https://doi.org/10.1038/s41392-020-00280-x. 
2. Morozov, B.S.; Namashivaya, S.S.R.; Zakharko, M.A.; Oshchepkov, A.S.; Kataev, E.A. Anthracene-Based Amido-Amine Cage Receptor for Anion Recognition under Neutral Aqueous Conditions. ChemistryOpen 2020, 9, 171-175, https://doi.org/10.1002/open.201900309.

3. Berhanu, A.L.; Gaurav; Mohiuddin, I.; Malik, A.K.; Aulakh, J.S.; Kumar, V.; Kim, K.-H. A review of the applications of Schiff bases as optical chemical sensors. TrAC, Trends Anal. Chem. 2019, 116, 74-91, https://doi.org/10.1016/j.trac.2019.04.025

4. Chakraborty, T.; Dasgupta, S.; Bhattacharyya, A.; Zangrando, E.; Escudero, D.; Das, D. A macrocyclic tetranuclear ZnII complex as a receptor for selective dual fluorescence sensing of F- and AcO-: effect of a macrocyclic ligand. New J. Chem. 2019, 43, 13152-13161, https://doi.org/10.1039/C9NJ03481A.

5. Hossain, M.S.; Roy, P.K.; Zakaria, C.M.; Zahan, M. Selected Schiff base coordination complexes and their microbial application: a review. Int. J. Chem. Stud 2018, 6, 19-31.

6. Al Zoubi, W.; Jirjees, V.; Suleman, V.; Al-Hamdani, A.A.S.; Ahmed, S.D.; Kim, Y.G.; Ko, Y.G. Synthesis and bioactivity studies of novel Schiff bases and their complexes. J. Phys. Org. Chem. 2019, 32, e4004, https://doi.org/10.1002/poc.4004.

7. Sadia, M.; Khan, J.; Naz, R.; Zahoor, M.; Khan, E.; Ullah, R.; Bari, A. Schiff-Based Fluorescent-ON Sensor L Synthesis and Its Application for Selective Determination of Cerium in Aqueous Media. Journal of Sensors 2020, 2020, 2192584, https://doi.org/10.1155/2020/2192584.

8. İnal, E.K. A Fluorescent Chemosensor Based on Schiff Base for the Determination of $\mathrm{Zn} 2+, \mathrm{Cd} 2+\mathrm{and} \mathrm{Hg} 2+$. Journal of Fluorescence 2020, 30, 891-900, https://doi.org/10.1007/s10895-020-02563-6.

9. Althobiti, H.A.; Zabin, S.A. New Schiff bases of 2-(quinolin-8-yloxy)acetohydrazide and their $\mathrm{Cu}(\mathrm{ii})$, and $\mathrm{Zn}$ (ii) metal complexes: their in vitro antimicrobial potentials and in silico physicochemical and pharmacokinetics properties. Open Chemistry 2020, 18, 591-607, https://doi.org/10.1515/chem-2020-0085.

10. O’Mullane D.M., Baez R.J., Jones S., Lennon M.A., Petersen P.E., RuggGunn A.J., Whelton H., Whitford G.M. Fluoride and Oral Health. Community Dental Health Journal. 2021; 38(1), 69-99, https://doi.org/10.1922/CDH_3707O’Mullane31.

11. Godebo, T.R.; Jeuland, M.; Tekle-Haimanot, R.; Shankar, A.; Alemayehu, B.; Assefa, G.; Whitford, G.; Wolfe, A. Bone quality in fluoride-exposed populations: A novel application of the ultrasonic method. Bone Reports 2020, 12, 100235, https://doi.org/10.1016/j.bonr.2019.100235.

12. Rosen, C.J. The Epidemiology and Pathogenesis of Osteoporosis. In Endotext, Feingold, K.R., Anawalt, B., Boyce, A., Chrousos, G., de Herder, W.W., Dhatariya, K., Dungan, K., Grossman, A., Hershman, J.M., Hofland, J., et al., Eds. MDText.com, Inc; 2020.

13. Coleman, R.; Hadji, P.; Body, J.J.; Santini, D.; Chow, E.; Terpos, E.; Oudard, S.; Bruland, Ø.; Flamen, P.; Kurth, A. Bone health in cancer: ESMO clinical practice guidelines. Ann. Oncol. 2020, 31, 1650-1663, https://doi.org/10.1016/j.annonc.2020.07.019

14. Ringe, J.D. Fluoride and Bone Health. In Nutrition and Bone Health, Holick, M.F., Dawson-Hughes, B., Eds. Humana Press: Totowa, NJ, 2004; https://doi.org/10.1007/978-1-59259-740-6_21.

15. Hattab, F.N. An update on fluorides and fluorosis with reference to oral health status in the gulf region. Asian Journal of Dental Sciences 2020, 27-48, https://www.journalajds.com/index.php/AJDS/article/view/30107.

16. Zhou, Y.; Wang, J.; Gu, Z.; Wang, S.; Zhu, W.; Acena, J.L.; Soloshonok, V.A.; Izawa, K.; Liu, H. Next Generation of Fluorine-Containing Pharmaceuticals, Compounds Currently in Phase II-III Clinical Trials of Major Pharmaceutical Companies: New Structural Trends and Therapeutic Area. Chem. Rev. 2016, 116, 422-518, https://doi.org/10.1021/acs.chemrev.5b00392.

17. Waugh, D.T. Fluoride Exposure Induces Inhibition of Sodium/Iodide Symporter (NIS) Contributing to Impaired Iodine Absorption and Iodine Deficiency: Molecular Mechanisms of Inhibition and Implications for Public Health. Int. J. Env. Res. Public Health 2019, 16, https://doi.org/10.3390/ijerph16061086.

18. Kurdi, M.S. Chronic fluorosis: The disease and its anaesthetic implications. Indian J. Anaesth. 2016, 60, 157, http://doi.org/10.4103/0019-5049.177867.

19. Deepika, R.M.S.; Nagaraj, S.K.; Sreeramulu, P.N. Mysterious Mineral: Fluoride. Journal of Evolution of Medical and Dental Sciences 2019, 8, 3617-3624, https://doi.org/10.14260/jemds/2019/781

20. Kheradpisheh, Z.; Mirzaei, M.; Mahvi, A.H.; Mokhtari, M.; Azizi, R.; Fallahzadeh, H.; Ehrampoush, M.H. Impact of Drinking Water Fluoride on Human Thyroid Hormones: A Case- Control Study. Sci. Rep. 2018, 8, 2674, https://doi.org/10.1038/s41598-018-20696-4.

21. Enderle, J.D. Chapter 8 - Biochemical Reactions and Enzyme Kinetics. In Introduction to Biomedical Engineering (Third Edition), Enderle, J.D., Bronzino, J.D., Eds. Academic Press: Boston, 2012; 447-508, https://doi.org/10.1016/B978-0-12-374979-6.00008-3. 
22. Maxwell, C.R.; Spangenberg, R.J.; Hoek, J.B.; Silberstein, S.D.; Oshinsky, M.L. Acetate Causes Alcohol Hangover Headache in Rats. PLoS One 2011, 5, e15963, https://doi.org/10.1371/journal.pone.0015963.

23. Martynov, V.I.; Pakhomov, A.A.; Popova, N.V.; Deyev, I.E.; Petrenko, A.G. Synthetic Fluorophores for Visualizing Biomolecules in Living Systems. Acta naturae 2016, 8, 33-46.

24. Lu, Y.; Zhang, E.; Yang, J.; Cao, Z. Strategies to improve micelle stability for drug delivery. Nano Research 2018, 11, 4985-4998, https://doi.org/10.1007/s12274-018-2152-3.

25. Okur, M.E.; Karantas, I.D.; Şenyiğit, Z.; Üstündağ Okur, N.; Siafaka, P.I. Recent trends on wound management: New therapeutic choices based on polymeric carriers. Asian Journal of Pharmaceutical Sciences 2020, 15, 661-684, https://doi.org/10.1016/j.ajps.2019.11.008.

26. Altay, Y.; Cao, S.; Che, H.; Abdelmohsen, L.K.E.A.; van Hest, J.C.M. Adaptive Polymeric Assemblies for Applications in Biomimicry and Nanomedicine. Biomacromolecules 2019, 20, 4053-4064, https://doi.org/10.1021/acs.biomac.9b01341.

27. Nakama, Y. Chapter 15 - Surfactants. In Cosmetic Science and Technology, Sakamoto, K., Lochhead, R.Y., Maibach, H.I., Yamashita, Y., Eds. Elsevier: Amsterdam, 2017; https://doi.org/10.1016/B978-0-12-802005$0.00015-\mathrm{X}$.

28. Stigter, D. Micelle formation by ionic surfactants. III. Model of Stern layer, ion distribution, and potential fluctuations. The Journal of Physical Chemistry 1975, 79, 1008-1014, https://doi.org/10.1021/j100577a013.

29. Sposito, G. Gouy-Chapman Theory. In Encyclopedia of Geochemistry: A Comprehensive Reference Source on the Chemistry of the Earth, White, W.M., Ed. Springer International Publishing: Cham, 2018, https://doi.org/10.1007/978-3-319-39312-4_50.

30. Quina, F.H.; Lissi, E.A. Photoprocesses in Microaggregates. Acc. Chem. Res. 2004, 37, 703-710, https://doi.org/10.1021/ar030152.

31. Ramamurthy, V.; Sivaguru, J. Supramolecular Photochemistry as a Potential Synthetic Tool: Photocycloaddition. Chem. Rev. 2016, 116, 9914-9993, https://doi.org/10.1021/acs.chemrev.6b00040.

32. Frisch, M.J.; Trucks, G.W.; Schlegel, H.B.; Scuseria, G.E.; Robb, M.A.; Cheeseman, J.R.; Scalmani, G.; Barone, V.; Mennucci, B.; Petersson, G.A.; Nakatsuji, H.; Caricato, M.; Li, X.; Hratchian, H.P.; Izmaylov, A.F.; Bloino, J.; Zheng, G.; Sonnenberg, J.L.; Hada, M.; Ehara, M.; Toyota, K.; Fukuda, R.; Hasegawa, J.; Ishida, M.; Nakajima, T.; Honda, Y.; Kitao, O.; Nakai, H.; Vreven, T.; Montgomery, J.A.; Peralta, Jr. J.A.; Ogliaro, F.; Bearpark, M.; Heyd, J.J.; Brothers, E.; Kudin, K.N.; Staroverov, V.N.; Kobayashi, R.; Normand, J.; Raghavachari, K.; Rendell, A.; Burant, J.C.; Iyengar, S.S.; Tomasi, J.; Cossi, M.; Rega, N.; Millam, J.M.; Klene, M.; Knox, J.E.; Cross, J.B.; Bakken, V.; Adamo, C.; Jaramillo, J.; Gomperts, R.; Stratmann, R.E.; Yazyev, O.; Austin, A.J.; Cammi, R.; Pomelli,R.; Ochterski, J.W.; Martin, R.L.; Morokuma, K.; Zakrzewski, V.G.; Voth, G.A.; Salvador, P.; Dannenberg, J.J.; Dapprich, S.; Daniels, A.D.; Farkas, O.; Foresman, J.B.; Ortiz, J.V.; Cioslowski, J.; Fox, D.J. Gaussian 09 Gaussian, Inc., Wallingford CT, 2009.

33. Rai, S.R.; Sarkar, A.; Pandey, A.; Dasgupta, S.; Majumder, I.; Das, D.; Bose, D.; Mukhopadhyay, M. Photometric Study of the Interaction of Zinc (II) Complexes of Schiff Bases with Cetyltrimethyl Ammonium Bromide. Macromolecular Symposia 2019, 388, 1900030, https://doi.org/10.1002/masy.201900030.

34. Benesi, H.A.; Hildebrand, J.H. A Spectrophotometric Investigation of the Interaction of Iodine with Aromatic Hydrocarbons. J. Am. Chem. Soc. 1949, 71, 2703-2707, https://doi.org/10.1021/ja01176a030.

35. Sen Chowdhury, M.; Sarkar, A.; Rai, S.R.; Dasgupta, S.; Majumder, I.; Bhattacharya, A.; Das, D.; Bose, D.; Mukhopadhyay, J.; Mukhopadhyay, M. Probing the binding interaction of zinc (II) Schiff bases with bovine serum albumin: A spectroscopic and molecular docking study. Appl. Organomet. Chem. 2021, 35, e6164, http://dx.doi.org/10.1002/aoc.6164. 